\title{
The Existence and Multiplicity of Solutions for Singular Boundary Value Systems with $p$-Laplacian
}

\author{
Zengxia Cai \\ School of Science, Linyi University, Linyi, China \\ Email: caizengxia64@163.com
}

Received January 2015

\section{Abstract}

This paper presents sufficient conditions for the existence of positive solutions for the fourth-order boundary value problem system with $p$-Laplacian operator. The existence of single or multiple positive solutions for the system is showed through the fixed point index theory in cones under some assumptions.

\section{Keywords}

\section{Coupled Singular Boundary Value Problem, Positive Solution, Fixed Point Index Theorem}

\section{Introduction}

In this paper, we are concerned with the existence and multiplicity of positive solutions for the system (BVP):

$$
\begin{cases}\left(\varphi_{p}\left(u^{\prime \prime}(t)\right)\right)^{\prime \prime}-a_{1}(t) f_{1}(u(t), v(t))=0, & 0<t<1, \\ \left(\varphi_{p}\left(v^{\prime \prime}(t)\right)\right)^{\prime \prime}-a_{2}(t) f_{2}(u(t), v(t))=0, \quad 0<t<1, & \\ u(0)=u(1)=u^{\prime \prime}(0)=u^{\prime \prime}(1)=0, & \\ v(0)=v(1)=v^{\prime \prime}(0)=v^{\prime \prime}(1)=0, & \end{cases}
$$

where $\varphi_{p}(s)=|s|^{p-2}, \quad s, p>1, \quad a_{i}(t) \in C((0,1),(0,+\infty))$ and $f_{i}(u, v) \in C([0,+\infty),[0,+\infty)), a_{i}(t)$ is allowed to have singularity at $t=0,1, i=1,2$.

Several papers ([1]-[4]) have studied the solution of fourth-order boundary value problems. But results about fourth-order differential eguations with $p$-Laplacian have rarely seen. Recently, several papers ([6]-[8]) have been devoted to the study of the coupled boundary value problem.

Motivated by the results mentioned above, here we establish some sufficient conditions for the existence of to (BVP) (1.1) under certain suitable weak conditions. The main results in this paper improve and generalize the results by others.

The following fixed-point index theorem in cones is fundamental. 
Theorem A [9] Assume that $X$ is a Banach space, $K \subseteq X$ is a cone in $X$, and $0<r<+\infty$, $\Omega_{r}=\{x \in k:\|x\| \leq r\}$, if $T: \Omega_{r} \mapsto X$ is a completely operator and $T x \neq x, \forall x \in \partial \Omega_{r}$.

1) If for $\forall u \in \partial \Omega_{r}, \quad\|x\| \leq\|T x\|$, then i $\left(T, \Omega_{r}, K\right)=0$;

2) If for $\forall u \in \partial \Omega_{r},\|x\| \geq\|T x\|$ then $\mathrm{i}\left(T, \Omega_{r}, K\right)=1$.

\section{Preliminaries and Lemmas}

In this paper, let $E=C[0,1]$ and $E^{+}=\{u \in E ; u(t) \geq 0$ is a concave function $\}$ then $E^{+} \times E^{+}$is a Banach space with the norm $\|(u, v)\|_{0}=\|u\|+\|v\|, \forall(u, v) \in E^{+} \times E^{+}$, where $\|u\|=\max _{0 \leq t \leq 1}\{u(t)\},\|v\|=\max _{0 \leq t \leq 1}\{v(t)\}$, then

$X:=E^{+} \times E^{+}$is a cone of $\left(E \times E\right.$, \|\|$\left._{0}\right)$. In tnhis paper, $\left(u_{1}, v_{1}\right) \geq\left(u_{2}, v_{2}\right)$ i.e. $u_{1} \geq u_{2}, v_{1} \geq v_{2}$

Suppose $G(t, s)$ is the Green function of the following boundary problem: $z=0,0<t<1, \quad z(0)=z(1)=0$, then

$$
G(t, s)= \begin{cases}s(1-t), & 0 \leq s \leq t \leq 1, \\ t(1-s), & 0 \leq t \leq s \leq 1,\end{cases}
$$

Obviously, $t(1-t) s(1-s) \leq G(t, s)=G(s, t) \leq t(1-t), \quad 0 \leq t, \quad s \leq 1$

Define a cone $K \subset X$ as follows $K=\left\{(u, v) \in X \mid(u, v) \geq(0,0), u(t)+v(t) \geq\|(u, v)\|_{0} t(1-t), t \in[0,1]\right\}$ and define an integral operator $A: K \rightarrow K$ by $A(u(t), v(t))=\left(A_{1}(u, v)(t), A_{2}(u, v)(t)\right)$, where

$$
A_{i}(u, v)(t)=\int_{0}^{1} G(t, s) \phi_{q}\left(\int_{0}^{1} G(s, \tau) a_{i}(\tau) f_{i}(u(\tau), v(\tau)) \mathrm{d} \tau\right) \mathrm{d} s, \quad i=1,2
$$

Let us list the following assumptions for convenience.

(H) $a_{i} \in C((0,1),[0,+\infty)), a_{i}(i=1,2)$ is singular at $t=0$ or 1 , and

$$
0<\int_{0}^{1} G(\tau, \tau) a_{i}(\tau) \mathrm{d} \tau<+\infty, \quad 0<\int_{0}^{1} G(s, \tau) a_{i}(\tau) \mathrm{d} \tau<+\infty, \quad i=1,2
$$

Lemma 2.1 $(u, v)$ is a solution of BVP (1.1) if and only if $(u, v) \in K, A(u, v)=(u, v)$ has fixed points.

It is easy to see that $(u, v) \in K, A(u, v)=(u, v)$ if $(u, v)$ is a solution of BVP (1.1).

Lemma 2.2 Suppose that $(H)$ hold, then $A K \subset K$.

Lemma 2.3 Suppose that $H$ hold. Then $A: K \rightarrow K$ is completely continuous.

Proof Firstly, assume $D \subset K$ is a bounded set, we have

$$
A_{i}(u, v)(t) \leq \int_{0}^{1} G(t, s) \phi_{q}\left(\int_{0}^{1} G(s, \tau) a_{i}(\tau)\right) \mathrm{d} s \phi_{q}\left(\sup f_{i}(u, v):(u, v) \in D\right)<+\infty
$$

Then $A_{i}(D)(i=1,2)$ is bounded, therefore $A(D)$ is bounded.

Secondly, suppose $\left(u_{n}, v_{n}\right),\left(u_{0}, v_{0}\right) \in D,\left(u_{n}, v_{n}\right) \rightarrow\left(u_{0}, v_{0}\right)(n \rightarrow \infty)$ then $\left(u_{n}, v_{n}\right)$ is bounded, we get

$$
\begin{aligned}
& \left|A\left(u_{n}, v_{n}\right)(t)-A\left(u_{0}, v_{0}\right)(t)\right| \\
& \leq \int_{0}^{1} G(s, s)\left|\phi_{q}\left(\int_{0}^{1} G(s, \tau) a_{1}(\tau) f_{1}\left(u_{n}, v_{n}\right)(\tau) \mathrm{d} \tau\right)-\phi_{q}\left(\int_{0}^{1} G(s, \tau) a_{1}(\tau) f_{1}\left(u_{0}, v_{0}\right)(\tau) \mathrm{d} \tau\right)\right| \mathrm{d} s \\
& \quad+\int_{0}^{1} G(s, s)\left|\phi_{q}\left(\int_{0}^{1} G(s, \tau) a_{2}(\tau) f_{2}\left(u_{n}, v_{n}\right)(\tau) \mathrm{d} \tau\right)-\phi_{q}\left(\int_{0}^{1} G(s, \tau) a_{2}(\tau) f_{2}\left(u_{0}, v_{0}\right)(\tau) \mathrm{d} \tau\right)\right| \mathrm{d} s \\
& \leq \frac{1}{4} \max _{0 \leq t \leq 1}\left|f_{1}^{q-1}\left(u_{n}(t), v_{n}(t)\right)-f_{1}^{q-1}\left(u_{0}(t), v_{0}(t)\right)\right| \phi_{q} \int_{0}^{1} \tau(1-\tau) a_{1}(\tau) \mathrm{d} \tau \\
& \quad+\frac{1}{4} \max _{0 \leq t \leq 1}\left|f_{2}^{q-1}\left(u_{n}(t), v_{n}(t)\right)-f_{2}^{q-1}\left(u_{0}(t), v_{0}(t)\right)\right| \phi_{q} \int_{0}^{1} \tau(1-\tau) a_{2}(\tau) \mathrm{d} \tau .
\end{aligned}
$$

Due to the continuity of $f_{1}, f_{2}$, by $H$ and above fomula together with Lebesgue Dominated Convergence Theorem, then $\left|A\left(u_{n}, v_{n}\right)(t)-A\left(u_{0}, v_{0}\right)(t)\right| \rightarrow 0$ when $n \rightarrow \infty$. Therefore $A$ is continuous.

Lastly, since $G(t, s)$ is continuous in $[0,1] \times[0,1]$, so it is uniformly continous. For all $\varepsilon>0, \exists \delta>0$ for all $s \in[0,1]$, when $\left|t_{1}-t_{2}\right|<\delta$, we get 


$$
\left|G\left(t_{1}, s\right)-G\left(t_{2}, s\right)\right|<\frac{\varepsilon}{2} \cdot\left\{\phi_{q}\left(\int_{0}^{1} G(\tau, \tau) a_{i}(\tau) \mathrm{d} \tau\right) \phi_{q}\left(\sup f_{i}(u, v):(u, v) \in D\right)\right\}^{-1}, \quad i=1,2
$$

Then for all $(u, v) \in D$, we have

$$
\begin{aligned}
\left|A(u, v)\left(t_{1}\right)-A(u, v)\left(t_{2}\right)\right| \leq & \int_{0}^{1}\left|G\left(t_{1}, s\right)-G\left(t_{2}, s\right)\right| \cdot \phi_{q}\left(\int_{0}^{1} G(\tau, \tau) a_{1}(\tau) \mathrm{d} \tau\right) \cdot \phi_{q}\left(\sup f_{1}(u, v):(u, v) \in D\right) \mathrm{d} s \\
& +\int_{0}^{1}\left|G\left(t_{1}, s\right)-G\left(t_{2}, s\right)\right| \cdot \phi_{q}\left(\int_{0}^{1} G(\tau, \tau) a_{2}(\tau) \mathrm{d} \tau\right) \cdot \phi_{q}\left(\sup f_{2}(u, v):(u, v) \in D\right) \mathrm{d} s \\
& <\frac{\varepsilon}{2}+\frac{\varepsilon}{2}=\varepsilon,
\end{aligned}
$$

So $A$ is equicontinuous, by Arzela-Ascoli theorem we know $A D$ is relatively compact.

Therefore, $A: \bar{K}_{r, R} \rightarrow K$ is completely continuous.

For convenience we denote

$$
\begin{aligned}
& f_{i 0}(u, v):=\varliminf_{u+v \rightarrow 0} \frac{f_{i}(u, v)}{(u+v)^{p-1}}, \quad f_{i \infty}(u, v):=\varliminf_{u+v \rightarrow 0} \frac{f_{i}(u, v)}{(u+v)^{p-1}}, \quad i=1,2 ; \\
& f_{i}^{0}(u, v):=\varlimsup_{u+v \rightarrow 0} \frac{f_{i}(u, v)}{(u+v)^{p-1}}, \quad f_{i}^{\infty}(u, v):=\varlimsup_{u+v \rightarrow 0} \frac{f_{i}(u, v)}{(u+v)^{p-1}}, \quad i=1,2 ; \\
& \mu_{i}:=\phi_{q}\left(\int_{0}^{1} G(\tau, \tau) a_{i}(\tau) \mathrm{d} \tau\right), \quad i=1,2 ; \\
& v_{i}:=\min _{\frac{1}{4} \leq t \leq \frac{1}{2}} \int_{\frac{1}{4}}^{\frac{1}{2}} G(t, s) \phi_{q}\left(\int_{0}^{1} G(s, \tau) a_{i}(\tau) \mathrm{d} \tau\right) \mathrm{d} s, \quad i=1,2 .
\end{aligned}
$$

\section{Main Results}

Theorem 3.1 Suppose that $H$ holds. If the following conditions are satisfied:

$$
\left(H_{1}\right) f_{i}^{0}(u, v)=0, \quad i=1,2 ;\left(H_{2}\right) \quad f_{1 \infty}(u, v)=\infty \text { or } f_{2 \infty}(u, v)=\infty
$$

Then the system (1.1) has at least one positive solution $(u(t), v(t)), t \in(0,1)$

Proof By Lemma 2.3, we know $A$ is completely continuous. By $\left(H_{1}\right)$, there exists $r_{1}>0$, when $0 \leq u(t)+v(t) \leq r_{1}, \quad t \in[0,1]$, we have $f_{i}(u, v) \leq\left(\alpha_{i}(u+v)\right)^{p-1}$, where $\alpha_{i}>0(i=1,2)$ satisfies $\max \left\{\alpha_{1} \mu_{1}, \alpha_{2} \mu_{2}\right\} \leq 3$. Let $\Omega_{r_{1}}=\left\{(u, v) \in K ;\|(u, v)\|_{0}<r_{1}\right\}$, when $(u, v) \in \partial \Omega_{r_{1}} \cap K$, we get

$$
A_{1}(u(t), v(t)) \leq \frac{\alpha_{1}}{6}\|u+v\| \phi_{q}\left(\int_{0}^{1} G(\tau, \tau) a_{1}(\tau) \mathrm{d} \tau\right) \leq \frac{\alpha_{1}}{6}\|(u, v)\|_{0} \mu_{1} \leq \frac{\|(u, v)\|_{0}}{2}
$$

Hence, $\left\|A_{1}(u, v)\right\| \leq \frac{\|(u, v)\|_{0}}{2}$. Similarly, we have $A_{2}(u(t), v(t)) \leq \frac{\|(u, v)\|_{0}}{2}$ then $\left\|A_{2}(u, v)\right\| \leq \frac{\|(u, v)\|_{0}}{2}$, therefore $\|A(u, v)\|_{0}=\left\{\left\|A_{1}(u, v)\right\|+\left\|A_{2}(u, v)\right\|\right\} \leq\|(u, v)\|_{0}, \quad \forall(u, v) \in \partial \Omega_{r_{1}} \cap K$. By Theorem A, i $\left(A, \Omega_{r_{1}} \cap K, K\right)=1$.

On the other hand, from $\left(H_{2}\right)$, if $f_{1 \infty}(u, v)=\infty$, there exists $R_{0}>r_{1}>0$, for $\beta_{1}>0$ satisfing $\beta_{1} v_{1} \geq 8$, we get $f_{1}(u, v) \geq\left(\beta_{1}(u+v)\right)^{p-1}$ when $u(t)+v(t) \geq R_{0}$. Set $R_{1}>R_{0}$ such that $R_{0} \leq\|u\|+\|v\| \leq R_{1}$, let $\Omega_{R_{1}}=\left\{(u, v) \in K ;\|(u, v)\|_{0}<R_{1}\right\}$, when $(u, v) \in \partial \Omega_{R_{1}} \cap K, t \in\left[\frac{1}{4}, \frac{1}{2}\right]$, we get $u+v \geq t(1-t)\|(u, v)\|_{0} \geq \frac{1}{8}\|(u, v)\|_{0}$, so

$$
A_{1}(u(t), v(t)) \geq \frac{\beta_{1}}{8}\|(u, v)\|_{0} \int_{\frac{1}{4}}^{\frac{1}{2}} G(t, s) \phi_{q}\left(\int_{0}^{1} G(s, \tau) a_{1}(\tau) \mathrm{d} \tau\right) \mathrm{d} s \geq \frac{\beta_{1}}{8}\|(u, v)\|_{0} v_{1} \geq\|(u, v)\|_{0}
$$


Hence, $\left\|A_{1}(u, v)\right\| \geq\|(u, v)\|_{0}$. then $\|A(u, v)\|_{0}=\left\|A_{1}(u, v)\right\|+\left\|A_{2}(u, v)\right\| \geq\|(u, v)\|_{0}, \quad \forall(u, v) \in \partial \Omega_{R_{1}} \cap K$

If $f_{2 \infty}(u, v)=\infty$, with the similar proofs of the condition $f_{1 \infty}(u, v)=\infty$, we get $\left\|A_{2}(u, v)\right\| \geq\|(u, v)\|_{0}$. Then $\|A(u, v)\|_{0}=\left\|A_{1}(u, v)\right\|+\left\|A_{2}(u, v)\right\| \geq\|(u, v)\|_{0}, \quad \forall(u, v) \in \partial \Omega_{R_{1}} \cap K$. In either case, we always may set $\|A(u, v)\|_{0} \geq\|(u, v)\|_{0}, \quad \forall(u, v) \in \partial \Omega_{R_{1}} \cap K \quad$ By Theorem A, $\mathrm{i}\left(A, \Omega_{R_{1}} \cap K, K\right)=0$ Through the additivity of the fixed point index we know that

$$
i\left(A,\left(\Omega_{R_{1}} \cap K\right) \backslash\left(\overline{\Omega_{r_{1}} \cap K}, K\right)\right)=i\left(A, \Omega_{R_{1}} \cap K, K\right)-i\left(A, \Omega_{r_{1}} \cap K, K\right)=0-1=-1
$$

Therefore it follows from the fixed-point theorem that $A$ has a fixed point $(u, v) \in\left(\Omega_{R_{1}} \cap K\right) \backslash\left(\overline{\Omega_{r_{1}} \cap K}\right)$, and thus $(u(t), v(t)), t \in(0,1)$ is a positive solution of BVP (1.1).

Theorem 3.2 Suppose that $H$ holds. If the following conditions are satisfied:

$$
\left(H_{3}\right) f_{i}^{\infty}(u, v)=0, \quad i=1,2 ;\left(H_{4}\right) f_{10}(u, v)=\infty \text { or } f_{20}(u, v)=\infty,
$$

Then the system (1.1) has at least one positive solution $(u(t), v(t)), t \in(0,1)$

Proof By lemma 2.3, we know $A$ is completely continuous. From $\left(H_{4}\right)$, if $f_{10}(u, v)=\infty$, for $\xi_{1}>0$ satisfying $\xi_{1} v_{1} \geq 8$, there exists $r_{1}^{\prime}>0$, when $0 \leq u(t)+v\left((t) \leq r_{1}^{\prime}, \quad t \in[0,1]\right.$, we have $f_{1}(u, v) \geq\left(\xi_{1}(u+v)\right)^{p-1}$. Let $\Omega_{r^{\prime}}=\left\{(u, v) \in K ;\|(u, v)\|_{0}<r_{1}^{\prime}\right\}$, when $(u, v) \in \partial \Omega_{r_{1}^{\prime}} \cap K, t \in\left[\frac{1}{4}, \frac{1}{2}\right]$, we get $u+v \geq t(1-t)\|(u, v)\|_{0} \geq \frac{1}{8}\|(u, v)\|_{0}$, then

$$
A_{1}(u(t), v(t)) \geq \frac{\xi_{1}}{8}\|(u, v)\|_{0} \int_{\frac{1}{4}}^{\frac{1}{2}} G(t, s) \phi_{q}\left(\int_{0}^{1} G(s, \tau) a_{1}(\tau) \mathrm{d} \tau\right) \mathrm{d} s \geq \frac{\xi_{1}}{8}\|(u, v)\|_{0} v_{1} \geq\|(u, v)\|_{0}
$$

Hence, $\left\|A_{1}(u, v)\right\| \geq\|(u, v)\|_{0}$. then $\|A(u, v)\|_{0}=\left\|A_{1}(u, v)\right\|+\left\|A_{2}(u, v)\right\| \geq\|(u, v)\|_{0}, \quad \forall(u, v) \in \partial \Omega_{r_{1}^{\prime}} \cap K$ If $f_{20}(u, v)=\infty$, take $\xi_{2}>0$ satisfying $\xi_{2} v_{2} \geq 8$, such taht $f_{2}(u, v) \geq\left(\xi_{2}(u+v)\right)^{p-1}$. Similarly, we get $\left\|A_{2}(u, v)\right\| \geq\|(u, v)\|_{0}$, then $\|A(u, v)\|_{0}=\left\|A_{1}(u, v)\right\|+\left\|A_{2}(u, v)\right\| \geq\|(u, v)\|_{0}, \forall(u, v) \in \partial \Omega_{r_{1}^{\prime}} \cap K \quad$ In either case, we always may set $\|A(u, v)\|_{0} \geq\|(u, v)\|_{0}, \forall(u, v) \in \partial \Omega_{r^{\prime}} \cap K$. By Theorem A, $\mathrm{i}\left(A, \Omega_{r_{1}^{\prime}} \cap K, K\right)=0$.

On the other hand, from $\left(H_{3}\right)$, there exists $R_{0}^{\prime}>r_{1}^{\prime}$ such that $f_{i}(u, v) \leq\left(\theta_{i}(u+v)\right)^{p-1}$, when $u+v \geq R_{0}$, where $\theta_{i}>0(i=1,2)$ satisfies $\max \left\{\theta_{1} \mu_{1}, \theta_{2} \mu_{2}\right\} \leq 3$. There are two cases to consider.

Case (i). Suppose that $\max _{1<\leq 1} f_{i}(u, v)(i=1,2)$ is bounded, then there exists $M_{i}>0$ satisfying $f_{i}(u, v) \leq M_{i}^{p-1}$, $i=1,2$. Taking $R_{1}^{\prime}>\max \left\{R_{0}^{\prime}, \frac{M_{1}}{3} \mu_{1}, \frac{M_{2}}{3} \mu_{2}\right\}$, let $\Omega_{R_{1}^{\prime}}=\left\{(u, v) \in K ;\|(u, v)\|_{0}<R_{1}^{\prime}\right\}$, when $(u, v) \in \partial \Omega_{R_{1}^{\prime}} \cap K$, we get

$$
A_{1}(u(t), v(t)) \leq \frac{M_{1}}{6} \phi_{q}\left(\int_{0}^{1} G(\tau, \tau) a_{1}(\tau) \mathrm{d} \tau\right) \leq \frac{\|(u, v)\|_{0}}{2}
$$

Hence, $\left\|A_{1}(u, v)\right\| \leq \frac{\|(u, v)\|_{0}}{2}$. Similarly, we have $A_{2}(u(t), v(t)) \leq \frac{\|(u, v)\|_{0}}{2}$, hence $\left\|A_{2}(u, v)\right\| \leq \frac{\|(u, v)\|_{0}}{2}$, then $\|A(u, v)\|_{0}=\left\|A_{1}(u, v)\right\|+\left\|A_{2}(u, v)\right\| \leq\|(u, v)\|_{0}, \quad \forall(u, v) \in \partial \Omega_{R_{1}^{\prime}} \cap K$.

Case (ii). Suppose that $\max _{1 \leq t \leq 1} f_{i}(u, v)(i=1,2)$ is unbounded, since $f_{i}(i=1,2)$ is continuous in $[0,+\infty) \times[0,+\infty)$, so there exists constant $R_{1}^{\prime} \geq R_{0}^{\prime}$ and two points $\left(u_{i}, v_{i}\right) \in[0,+\infty) \times[0,+\infty)$ such that $R_{0}^{\prime} \leq u_{i}+v_{i} \leq R_{1}^{\prime}$, and $f_{i}(u, v) \leq f_{i}\left(u_{i}, v_{i}\right)$. Then we get $f_{i}(u, v) \leq f_{i}\left(u_{i}, v_{i}\right) \leq\left(\theta_{i}\left(u_{i}+v_{i}\right)\right)^{p-1} \leq\left(\theta_{i} R_{1}^{\prime}\right)^{p-1}, i=$ 1

2. Let $\Omega_{R_{1}^{\prime}}=\left\{(u, v) \in K ;\|(u, v)\|_{0}<R_{1}^{\prime}\right\}$, when $(u, v) \in \partial \Omega_{R_{1}^{\prime}} \cap K$, we get

$$
A_{1}(u(t), v(t)) \leq \frac{\theta_{1} R_{1}^{\prime}}{6} \phi_{q}\left(\int_{0}^{1} G(\tau, \tau) a_{1}(\tau) \mathrm{d} \tau\right) \leq \frac{R_{1}^{\prime}}{2}=\frac{\|(u, v)\|_{0}}{2}
$$


Hence, $\left\|A_{1}(u, v)\right\| \leq \frac{\|(u, v)\|_{0}}{2}$. Similarly, we have $A_{2}(u(t), v(t)) \leq \frac{R_{2}^{\prime}}{2}=\frac{\|(u, v)\|_{0}}{2}$, then $\left\|A_{2}(u, v)\right\| \leq \frac{\|(u, v)\|_{0}}{2}$, so $\|A(u, v)\|_{0}=\left\|A_{1}(u, v)\right\|+\left\|A_{2}(u, v)\right\| \leq\|(u, v)\|_{0}, \forall(u, v) \in \partial \Omega_{R_{1}^{\prime}} \cap K$. In either case, we always may set $\|A(u, v)\|_{0} \leq\|(u, v)\|_{0}, \forall(u, v) \in \partial \Omega_{R_{1}^{\prime}} \cap K$. By Theorem A, $\mathrm{i}\left(A, \Omega_{R_{1}^{\prime}} \cap K, K\right)=1$. Through the additivity of the fixed point index we know that

$$
i\left(A,\left(\Omega_{R_{1}^{\prime}} \cap K\right) \backslash\left(\overline{\Omega_{r_{1}^{\prime}} \cap K}, K\right)\right)=i\left(A, \Omega_{R_{1}^{\prime}} \cap K, K\right)-i\left(A, \Omega_{r_{1}^{\prime}} \cap K, K\right)=0-1=-1
$$

Therefore it follows from the fixed-point theorem that $A$ has a fixed point $(u, v) \in\left(\Omega_{R_{1}^{\prime}} \cap K\right) \backslash\left(\overline{\Omega_{r_{1}^{\prime}} \cap K}\right)$, and thus $(u(t), v(t)), t \in(0,1)$ is a positive solution of BVP (1.1). This completes the proof.

Remark 3.1 Note that if $f$ is superlinear or sublinear, our conclusions hold. Limit conditions of $f$ in this paper are more weak and general.

Remak 3.2 When $u=v, f_{1}=f_{2}$ and $w_{1}=w_{2}$, our results generalize and improve the results of [1]-[4].

Theorem 3.3 Suppose that $H$ holds. If the following conditions are satisfied:

$\left(H_{5}\right) f_{i}^{0}(u, v)=\rho_{i} \in[0,+\infty), i=1,2$ where $\rho_{i}(i=1,2)$ satisfies $\max \left\{\rho_{1}^{q-1} \mu_{1}, \rho_{2}^{q-1} \mu_{1}\right\} \leq 3$;

$\left(H_{6}\right) f_{1 \infty}(u, v)=\lambda_{1} \in(0,+\infty)$ or $f_{2 \infty}(u, v)=\lambda_{2} \in(0,+\infty)$, where $\lambda_{i}$ satisfies $\lambda_{i}^{q-1} v_{i} \geq 8 \quad(i=1$ or $i=2)$ then the system (1.1) has at least one positive solution $(u(t), v(t)), \quad t \in(0,1)$

Proof. Choosing $\varepsilon_{i}>0(i=1,2)$ such that $\max \left\{\left(\rho_{1}+\varepsilon_{1}\right)^{q-1} \mu_{1},\left(\rho_{2}+\varepsilon_{2}\right)^{q-1} \mu_{2}\right\} \leq 3$ and $\left(\lambda_{i}-\varepsilon\right)^{q-1} v_{i} \geq 8$, $i=1$ or $i=2$ From $\left(H_{5}\right)$, there exists $r_{1}^{*}>0$ such that $f_{i}(u, v) \leq\left(\rho_{i}+\varepsilon_{i}\right)(u, v)^{p-1} \quad(i=1,2)$ when $0 \leq u+v \leq r_{1}^{*}$. Let $\Omega_{r_{1}^{*}}=\left\{(u, v) \in K ;\|(u, v)\|_{0}<r_{1}^{*}\right\}$ when $(u, v) \in \partial \Omega_{r_{1}^{*}}^{*} \cap K$, we get

$$
A_{1}(u(t), v(t)) \leq \frac{\left(\rho_{1}+\varepsilon_{1}\right)^{q-1}}{6}\|u+v\| \phi_{q}\left(\int_{0}^{1} G(\tau, \tau) a_{1}(\tau) \mathrm{d} \tau\right) \leq \frac{\left(\rho_{1}+\varepsilon_{1}\right)^{q-1}}{6}\|(u, v)\|_{0} \mu_{1} \leq \frac{\|(u, v)\|_{0}}{2}
$$

Hence, $\left\|A_{1}(u, v)\right\| \leq \frac{\|(u, v)\|_{0}}{2}$. Similarly, we have $A_{2}(u(t), v(t)) \leq \frac{\|(u, v)\|_{0}}{2}$, so $\left\|A_{2}(u, v)\right\| \leq \frac{\|(u, v)\|_{0}}{2}$, then $\|A(u, v)\|_{0}=\left\|A_{1}(u, v)\right\|+\left\|A_{2}(u, v)\right\| \leq\|(u, v)\|_{0}, \quad \forall(u, v) \in \partial \Omega_{r_{1}^{*}} \cap K$. By Theorem A, i $\left(A, \Omega_{r_{1}^{*}} \cap K, K\right)=1$.

On the other hand, From $\left(H_{6}\right)$, if $f_{1 \infty}(u, v)=\lambda_{1}$, there exists $R_{0}^{*}>r_{1}^{*}$, such that $f_{1}(u, v) \geq\left(\lambda_{1}-\varepsilon_{1}\right)(u, v)^{p-1}$ when $u(t)+v(t) \geq R_{0}^{*}$. Set $R_{1}^{*}>R_{0}^{*}$ such that $R_{0} \leq\|u\|+\|v\| \leq R_{1}^{*}$, let

$\Omega_{R_{1}}=\left\{(u, v) \in K ;\|(u, v)\|_{0}<R_{1}^{*}\right\}$, when $(u, v) \in \partial \Omega_{R_{1}^{*}} \cap K, t \in\left[\frac{1}{4}, \frac{1}{2}\right]$, we get $u+v \geq t(1-t)\|(u, v)\|_{0} \geq \frac{1}{8}\|(u, v)\|_{0}$, then

$$
\begin{aligned}
A_{1}(u(t), v(t)) & \geq \frac{\left(\lambda_{1}-\varepsilon_{1}\right)^{q-1}}{8}\|(u, v)\|_{0} \int_{\frac{1}{4}}^{\frac{1}{2}} G(t, s) \phi_{q}\left(\int_{0}^{1} G(s, \tau) a_{1}(\tau) \mathrm{d} \tau\right) \mathrm{d} s \\
& \geq \frac{\left(\lambda_{1}-\varepsilon_{1}\right)^{q-1}}{8}\|(u, v)\|_{0} v_{1} \geq\|(u, v)\|_{0},
\end{aligned}
$$

Hence, $\left\|A_{1}(u, v)\right\| \geq\|(u, v)\|_{0}$. then $\|A(u, v)\|_{0}=\left\|A_{1}(u, v)\right\|+\left\|A_{2}(u, v)\right\| \geq\|(u, v)\|_{0}, \quad \forall(u, v) \in \partial \Omega_{R_{1}^{*}} \cap K$.

If $f_{2 \infty}(u, v)=\lambda_{2}$, by $\left(\lambda_{2}-\varepsilon\right)^{q-1} v_{2} \geq 8$, with the similar proofs of the condition $f_{100}(u, v)=\lambda_{1}$, we get $\left\|A_{2}(u, v)\right\| \geq\|(u, v)\|_{0}$. Then $\|A(u, v)\|_{0}=\left\|A_{1}(u, v)\right\|+\left\|A_{2}(u, v)\right\| \geq\|(u, v)\|_{0}, \forall(u, v) \in \partial \Omega_{R_{1}^{*}} \cap K \quad$ In either case, we always may set $\|A(u, v)\|_{0} \geq\|(u, v)\|_{0}, \forall(u, v) \in \partial \Omega_{R_{1}^{*}} \cap K$. By Theorem A, i $\left(A, \Omega_{R_{1}^{*}} \cap K, K\right)=0$. Through the additivity of the fixed point index we know that

$$
i\left(A,\left(\Omega_{R_{1}^{*}} \cap K\right) \backslash\left(\overline{\Omega_{r_{1}^{*}} \cap K}, K\right)\right)=i\left(A, \Omega_{R_{1}^{*}} \cap K, K\right)-i\left(A, \Omega_{r_{1}^{*}} \cap K, K\right)=0-1=-1
$$

Therefore it follows from the fixed-point theorem that $A$ has a fixed point $(u, v) \in\left(\Omega_{R_{1}^{*}} \cap K\right) \backslash\left(\overline{\Omega_{r_{1}^{*}} \cap K}\right)$, and thus $(u(t), v(t)), \quad t \in(0,1)$ is a positive solution of BVP (1.1). This completes the proof.

Theorem 3.4 Suppose that $H$ holds. If the following conditions are satisfied: 
$\left(H_{7}\right) f_{i 0}(u, v)=\rho_{i}^{*} \in(0,+\infty), i=1$ or $i=2$ where $\rho_{i}^{*}(i=1,2)$ satisfies $\rho_{i}^{q-1} v_{i} \geq 8, \quad(i=1$ or $i=2)$;

$\left(H_{8}\right) f_{i}^{\infty}(u, v)=\lambda_{i}^{*} \in[0,+\infty)$ where $\lambda_{i}^{*}$ satisfies $\max \left\{\lambda_{1}^{q-1} \mu_{1}, \lambda_{2}^{q-1} \mu_{2}\right\} \leq 3, \quad(i=1,2)$, then the system

(1.1) has at least one positive solution $(u(t), v(t)), t \in(0,1)$

The proofs are similar to that of Theorem 3.2 and are omitted.

Theorem 3.5 Assume that $\mathrm{H}, \mathrm{H}_{2}$ holds. If the following conditions are satisfied:

$$
\left(H_{9}\right) f_{i 0}(u, v)=0, \quad i=1,2 ;\left(H_{10}\right) f_{1}^{0}(u, v)=\infty \text { or } f_{2}^{0}(u, v)=\infty,
$$

Then the system (1.1) has at least two positive solutions $\left(u_{1}(t), v_{1}(t)\right)$ and $\left(u_{2}(t), v_{2}(t)\right)$ satisfying $0<\left\|\left(u_{1}, v_{1}\right)\right\|_{0} \leq r_{1} \leq\left\|\left(u_{2}, v_{2}\right)\right\|_{0}$.

Theorem 3.6 Assume that $H, H_{3}, H_{9}, H_{10}$ hold. then the system (1.1) has at least two positive solutions $\left(u_{1}(t), v_{1}(t)\right)$ and $\left(u_{2}(t), v_{2}(t)\right)$ satisfying $0<\left\|\left(u_{1}, v_{1}\right)\right\|_{0} \leq r_{1} \leq\left\|\left(u_{2}, v_{2}\right)\right\|_{0}$.

Remark 3.3 Under suitable weak conditions, the multiplicity results for fourth-order singular boundary value problem with $p$-Laplacian are established. Our results extend and improve the results of [5]-[8].

\section{References}

[1] Korman, P. (2004) Uniqueness and Exact Multiplicity of Solutions for a Class of Fourth-Order Semilinear Problems. Proceedings of the Royal Society of Edinburgh Section A-Mathematics, A134, 179-190. http://dx.doi.org/10.1017/S0308210500003140

[2] Ma, R. and Wu, H. (2002) Positive Solutions of a Fourth-Order Two-Point Boundary Value Problem. Acta Mathematica Sinica, A22, 244-249. (In Chinese)

[3] Yao, Q. (2004) Positive Solutions for Eigenvalue Problems of Fourth-Order Elastic Beam Equations. Applied Mathematics Letters, 17, 237-243. http://dx.doi.org/10.1016/S0893-9659(04)90037-7

[4] Ma, R.Y. and Wang, H.Y. (1995) On the Existence of Positive Solutions of Fourth-Order Ordinary Differential Equations. Applicable Analysis, 59, 225-231. http://dx.doi.org/10.1080/00036819508840401

[5] Sun, W.P. and Ge, W.G. The Existence of Positive Solutions for a Class of Nonlinear Boundary Value Problems. Acta Mathematica Sinica, 44, 577-580. (In Chinese)

[6] Agarwal, R.P., O’Regan, D. and Wong, P.J. (2000) Positive Solutions of Differential. Difference and Integral Equations. Springer-Verlag, Singapore.

[7] Ma, R.Y. (2000) Multiple Nonnegative Solutions of Second-Order Systems of Boundary Value Problems. Nonlinear Analysis, 42, 1003-1010. http://dx.doi.org/10.1016/S0362-546X(99)00152-2

[8] Ni, X.H. and Ge, W.G. (2005) Existence of Positive Solutions for One-Dimensional p-Laplacian Coupled Boundary Value Problem. J. Math. Rese. Expo., 25, 489-494. (In Chinese)

[9] Guo, D.J. (2000) Nonlinear Functional Analysis. Science and Technology, Jinan. 